\title{
Explore the Different Performance of Epidemic Prevention and Control: Through the Mutual Exchange of Health QR Code
} -- Based on the Theoretical Perspective of Government Management Mode

\author{
Jingheng $\mathrm{Xu}^{1, *}$

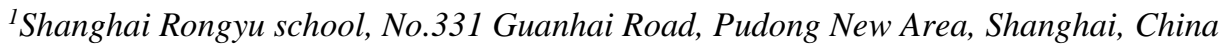 \\ * Jingheng Xu. Email: geography0107@gmail.com
}

\begin{abstract}
During the prevention and control of COVID-19, various provinces in China have innovatively introduced unique health QR codes covering their own provinces, among which Jiangsu Province is the most special one. Although Jiangsu Province launched the "Jiangsu Health Code" in March and promoted the mutual recognition of health QR code within the province, its 13 prefecture-level cities continued to retain the original local health QR codes, which was truly unified until July 24, more than half a year after the outbreak of the epidemic. By focusing on the large degree of dispersion in health QR codes in Jiangsu province, this paper will state the underlying causes and propose possible explanations. In addition, by comparing COVID-19 prevention and control data, this paper will analyze the effective control of the epidemic spread in Jiangsu Province. This seemingly decentralized governance model of local governments in Jiangsu is more effective than those in other provinces with vertical management and follow the unified regulation of the provincial government. Therefore, the author draws the following conclusion in the paper that the flat government management model is more advantageous than the vertical management mode in the prevention and control of public health security emergencies in specific regions.
\end{abstract}

Keywords: Epidemic Prevention, Mutual Exchange, Health QR Code, Government Management

\section{INTRODUCTION}

Since the outbreak of the novel coronavirus at the beginning of this year, various provinces and cities across China have innovatively introduced local "health QR codes," which helps better to curb the spread and diffusion of the novel coronavirus, strengthen community management, and steadily promote the resumption of production and rehabilitation, to make the prevention and control the outbreak becomes more precise, scientific and orderly. All parts of China have innovatively introduced local "health QR codes" as an essential safety prerequisite for everyday life. People only need to declare their recent travel routes online. Whether they have close contact history with infected people and whether they have a physical deficiency and fever symptoms, etc. After the system's background review, they can generate a personal health $\mathrm{QR}$ code. This health QR code can divide the individual's risk of infection into high risk, medium risk, and low risk, corresponding to the red, yellow, and green displayed respectively. Through the implementation of the innovative measures of "red code forbidden to go out, yellow code for restricted going out, and green code for normal going out," the possibility of epidemic transmission is minimized to effectively improve the convenience for Chinese people to carry out everyday life and work under the epidemic background and the management efficiency of the government's normalization of epidemic prevention and control. "Preparing personal health QR code on the mobile phone before going out" has become one of the simplest Chinese people's habits during this pandemic, which can be seen that this simple and straightforward health QR code has played an indelible role in China's fight against COVID-19. 
Jiangsu province, with the second-largest GDP and the highest population density in China, will inevitably be the first to launch a local health $\mathrm{QR}$ code during the fight against the epidemic due to the massive demand of its industries and workers for returning to work and production. The facts are accurate. However, different from other provinces (for example, Zhejiang province implements the "Zhekang health QR code" and Anhui province implements the "Ankang health QR code"), despite the existence of the unified health QR code "Jiangsu Health Code" in Jiangsu Province, the 13 prefecture-level cities in Jiangsu Province still insist on their health QR code, which is not connected with other counties and towns in the province. For example, Xuzhou is "Pengcheng code," Nantong is "Yilaitong code," Nanjing is "Ningguilai code," Wuxi is "Xikang code," Suzhou is "Sucheng code," Zhenjiang is "Zhen health code," and so on. This style and practice, which is entirely different from other regions, once became a hot search on Weibo during the epidemic, causing widespread concern and discussion from all walks of life. "One code for one city" is common ridicule by many netizens on the phenomenon that the cities in Jiangsu Province are in charge of each other and ignore the provincial government's unified command.

In the dictionary of modern national governance, flat management mode (also called decentralized management) refers to a compact and efficient flat organizational structure with multiple institutions or individuals arranged in parallel and without affiliation. The above description is very consistent with the local government's performance in Jiangsu Province in the process of fighting the epidemic, which is managed independently of the provincial government. On the contrary, the government vertical management mode (also known as centralized management) means that the central and provincial governments directly manage some administrative units and departments, and the personnel of these units should strictly implement the management system directly appointed by the central or provincial government (Zhou Quan, 2009). In the vertical organizational structure, the subordinate departments only accept the instructions from one superior, and the person in charge at all levels should be responsible for all the problems in their subordinate units. Take Shandong Province as an example. In the process of COVID-19 prevention and control, 17 cities in Shandong have always maintained the accurate unification and implementation of policies issued by the provincial government, which conforms to the above description of vertical management mode. To sum up, Jiangsu and Shandong have adopted two completely different approaches to the prevention and control of COVID-19.

Moreover, by comparing the prevention and control results of the COVID-19 epidemic in Shandong and Jiangsu provinces (combined with the infection rate per million population, the date of the release of tertiary and secondary response by the province, etc.), it can be seen that the prevention and control results of Jiangsu are significantly better than those of Shandong. Jiangsu Province has been reduced from the first level of the COVID-19 epidemic prevention and control response to the second level on February 24, and Shandong Province made corresponding adjustments 13 days later. Besides, Jiangsu Province lowered its response level from the second level to the third level as early as March 27, while Shandong province waited until May 6, more than a month later than Jiangsu province.

Table1. Comparison of the releasing date of prevention and control in the two provinces

\begin{tabular}{|l|l|l|}
\hline & $\begin{array}{l}\text { Jiangsu } \\
\text { province }\end{array}$ & $\begin{array}{l}\text { Shandong } \\
\text { province }\end{array}$ \\
\hline $\begin{array}{l}\text { Date of } \\
\text { releasing first- } \\
\text { level prevention }\end{array}$ & February 24 & March 8 \\
\hline $\begin{array}{l}\text { Date of } \\
\text { releasing } \\
\text { second-level } \\
\text { prevention }\end{array}$ & March 27 & May 6 \\
\hline
\end{tabular}

Only from the point of time to release the response level it can be seen that the decentralized governance model in Jiangsu, which seems to be in charge of local governments, is more effective in controlling the spread of the virus than other provinces (such as Shandong) which have vertical management and follow the unified scheduling of the provincial government. The research in this article will contribute to the discussion of which management system is more beneficial to respond to emergencies and provide reference information and new thinking angles for China to deal with the current COVID-19 epidemic and future social governance.

\section{RELEVANT LITERVATURE REVIEW OF GOVERNANCE MODEL}

For a long time, vertical and decentralized government models, which of these two governance frameworks can operate most effectively and better, has always been a hot topic in the academic circles and public policy-making fields. As well as their causes, the specific mode of operation and the resulting consequences have been the focus of academic debate.

Over the past 70 years, China has also changed and adjusted its government governance model for different development stages. In the middle of the 20th century, when the People's Republic of China was founded, the Central Committee of the CPC formed a highly centralized political system to consolidate the new regime and strengthen the central administrative units' unity and authority law enforcement. However, with the completion of socialism's fundamental transformation, this vertical political system's disadvantages began to 
emerge gradually. Especially after the reform and opening up in the late 1970s, local protectionism intensified with the deepening of economic reform. With the division of local agencies and officials, the Party Central Committee lost its independent power and status, and there was a phenomenon of vertical management failure. Since the reform of the market economy can only be effectively implemented under the unified national policy, the central government decided to withdraw some administrative power to achieve the goal of policy smoothness and reduce local governments' interference.

In his empirical analysis "Regulating the Relationship between Vertical Management Departments and Local Governments," Zhou Quan pointed out that the vertical management system is conducive to the rational allocation of resources, reduces the local protectionism that divides the market, ensures the smoothness of the central government's orders, and has the advantages of truthfully reflecting the situation of the basic statistics [7]. Besides, there are many scholars including Zhou Quan and Huang Tao, such as $\mathrm{Li}$ Yichun and $\mathrm{Wu}$ Baiyi not only pointed out the causes of localization of vertical management mode but also showed its hazards, reminding the central government to be vigilant against this localization phenomenon [2][5]. There are three central arguments in their article, which can be roughly summarized as follows. First, if the vertical management sector is localized, it is evident that national unity and development will be seriously harmed, because local protectionism will divide the interests of a larger area and even the development of the country. Second, after the localization of vertical management departments, the functions of local organizations at all levels will be incomplete, and the enthusiasm of local work and policy coordination with the central government will be seriously affected. Third, the personnel rights and financial rights of the vertical management departments are decided by the higher authorities in the system, and are not subject to the supervision of other judicial departments. Too much localized management will inevitably lead to the reduction of supervision departments, which will easily breed official embezzlement and corruption, and lead to the regulation of departmental interests.

At present, domestic research on government vertical management model is very thorough, mainly including the following three aspects, the cause of the model, how to improve to adapt to different development needs and avoid localization of management department. Many scholars have also made many useful explorations for the improvement of social governance models and the future direction of national governance. But so far, the author has found only a few in-depth studies on the advantages of flat local government.
Among them, $\mathrm{Wu}$ Baiyi believes that scholars at home and abroad have limitations and one-sidedness in studying China's development achievements. They tend to focus on the power efficiency model, but ignore that this model has run into a huge obstacle -- vested interests. He cites the most significant corruption cases of the past decade and the development paths of South Korea and Japan since the 1970s. Excessive concentration of institutional power will lead to severe inequality and inequality in distribution and ultimately lead to depleting the endogenous vitality of development. Wu Baiyi finally concluded that under the leadership of the party, China should break the old power efficiency model, decentralize power to local governments, modestly learn from the flat social governance of other countries, such as Germany and Nordic countries, and constantly improve and promote modern national governance to distribute further the dividends of reform and opening up [5].

Li Yichun also quoted the case of General Electric Company in the United States and the research results of foreign professors, and put forward the following views: The main body of social management should be the benign interaction structure between individual citizens, autonomous citizen organizations, social organizations (social groups, private non-enterprises) and government public power, which shows that the future social governance model should embody the spirit of flattening, pluralism, cooperation and democratic consultation [2].

However, most of the existing research on flat social governance is limited to promoting economic development, production efficiency, and strengthening social cohesion but lack analysis of the causes of decentralized power governance mode. In particular, only a few mathematicians have explored its role and specific effect in dealing with significant crises (such as epidemic situation and economic crisis, etc.), which can achieve the same or even surpass the impact of centralized management. These studies prove that the academic community has a stereotype of this term, believing that it is not convenient for unified dispatch and management after the decentralization of powers to local governments, thus hindering the implementation of policies and regional development.

In this article, the author will compare the two provinces of Shandong and Jiangsu, which are also under China's political climate, to analyze and study the different response styles and governance methods under the outbreak of COVID-19. In this article, the author chooses to discuss which model performs better in dealing with crises from an entirely different perspective. The data used in this article are from the first half of 2020, when the novel coronavirus just broke out in China. Based on this case study, the authors demonstrate that decentralized government management 
is more favorable than centralized management to prevent and control public health security emergencies in specific regions.

\section{GENERAL SITUATION AND EPIDEMICPREVENTION AND CONTROL PERFORMANCE OF JIANGSU PROVINCE}

\subsection{General situation of the development of Jiangsu Province}

Before exploring the decentralized epidemic control in Jiangsu Province, by citing the previous literature, the author would like to briefly discuss Jiangsu province's development situation and the specific reasons for "prefecture-level infighting," which is rarely seen in other regions of China.

Jiangsu province is located in China's southeast coastal area, covering more than 100,000 square kilometers, with the permanent resident population ranking fifth in China. The level of economic development of Jiangsu province has reached the status of the generally developed countries. In addition, its livelihood index, regional development index, and per capita GDP all rank first in the national economic index. Usually, according to the past economic development and different cultural and historical areas, people will divide Jiangsu province into three regions: Southern Jiangsu, Central Jiangsu, and Northern Jiangsu. Discussions on the gap in Jiangsu Province will also be based on this assumption.

As a matter of fact, many readers who pay attention to news should be familiar with the phenomenon of independence and rivalry among counties and cities in Jiangsu Province. By searching for the previous literature, we find that many authors have written articles pointing out the economic and cultural differences within Jiangsu Province and their causes and countermeasures.

First of all, we can see the difference in Jiangsu province from the data. In the GDP report of Jiangsu Province in 2013, the five prefecture-level cities south of the Yangtze River accounted for 3.638568 billion yuan, accounting for $59 \%$ of the total amount province. In contrast, even though the eight prefecture-level cities north of the Yangtze River have a large area, the real GDP only accounts for $41 \%$ of the province. Also, citing the 2019 China GDP per capita report, the gap between Suzhou and Lianyungang, the No.1, is nearly three times the lowest in Jiangsu province. In the latest list of the top 100 private enterprises in Jiangsu, 76 companies in Southern Jiangsu are listed. Despite the vast area of northern Jiangsu, only 24 companies are on the list. Finally, let's take a look at the general situation of investment projects in Jiangsu Province in 2017. There are 667 projects in the five cities in Southern
Jiangsu, with a total investment of 797.3 billion yuan, accounting for $60 \%$. Among them, Nanjing has the most extensive project scale in the province, reaching 271.6 billion yuan. The number of projects started in Suzhou has reached 177, and the annual investment has reached 59.4 billion yuan. There were 676 projects in eight cities of Central Jiangsu, with a total investment of 534.4 billion yuan, accounting for $40 \%$.

Table2. Comparison of the gap data in Jiangsu Province

\begin{tabular}{|l|l|l|}
\hline & $\begin{array}{l}\text { Eight cities in } \\
\text { Northern } \\
\text { Jiangsu }\end{array}$ & $\begin{array}{l}\text { Five towns in } \\
\text { Southern } \\
\text { Jiangsu }\end{array}$ \\
\hline Land area & $72.83 \%$ & $27.17 \%$ \\
\hline $\begin{array}{l}2013 \text { GDP } \\
\text { statistics }\end{array}$ & $41 \%$ & $59 \%$ \\
\hline $\begin{array}{l}\text { Top 100 private } \\
\text { enterprises in } \\
\text { Jiangsu province }\end{array}$ & $\begin{array}{l}24 \\
\text { enterprises }\end{array}$ & 76 enterprises \\
\hline $\begin{array}{l}\text { Investment } \\
\text { situation of } \\
\text { Jiangsu Province } \\
\text { in } 2017\end{array}$ & $40 \%$ & $60 \%$ \\
\hline
\end{tabular}

From the perspective of history and culture, the author, as a native of Jiangsu, is well aware of the differences in cities' customs and habits in the province. There are three dialect regions in Jiangsu Province: Zhongyuan dialect, Jianghuai dialect, and Wu Dialect. Therefore, the author strongly agrees with the discussion of scholars Miao Kai and Hu Jing in the article "Cultural Interpretation of Southern Jiangsu Development" [3]: "The regional culture of Jiangsu is divided into three cultural areas: Wu Cultural Area, Chu-Han cultural Area and Huaiyang cultural Area. These three regional cultures have their advantages, but there are also some limitations. Due to the overemphasis on seeking progress instability, there are too many cautious elements in $\mathrm{Wu}$ culture. The lack of adventurous spirit in economic development is also due to the strong local consciousness. Simultaneously, the ancient hierarchical system and the wind of maintaining the existing order in Southern Jiangsu are continuing to this day to a large extent. For an extended period, it is easy to develop official oriented consciousness and lack management innovation. While the Chu-Han culture is orthodox, feudal, and obstinate, Chu-Han culture is more honest than flexible, keeping to the old ways, hindering people's acceptance of advanced civilization and new things, and restricting the growth of talents, enthusiasm, and creativity. The shortcomings of Huaiyang culture are also apparent. For example, Jinling culture has evident dependence on foreign countries and 
fragility of development, Huaiyang culture is closed and conservative, Haiyan culture is not open enough, and it is more secured and conservative. Finally, Miao Kai and Hu Jing (Miao Kai; Hu Jing, 2008) concluded that such regional cultural differences in Jiangsu province made it unbalanced in economic and social development, and globalization and marketization further increased the cultural gap, which could only be adjusted through the construction of core values of socialism.

In summary, due to the cultural influence formed by different histories, coupled with the existence of the Yangtze River, Huaihe River, and other natural obstacles and policy reasons, the additional development paths and levels of cities in the province are very different. Furthermore, the dialects, eating habits, economic system and other aspects of Jiangsu Province are also very different. Apart from geographical proximity, there are not many similar features, more like a forced patchwork. Despite this, all 13 cities in Jiangsu Province are among the top 100 Chinese cities in GDP, unique among many provinces in China. The relatively balanced economic development and the particular historical culture of each region make it difficult for Jiangsu Province to produce a "provincial capital" that genuinely has absolute leadership in all aspects. Therefore, it is not difficult to understand the unique phenomenon that the 13 cities in Jiangsu province each had their own independent prevention and control policies and health QR codes during the fight against the COVID-19 epidemic at the beginning of this year.

\subsection{Epidemic prevention and control performance of Jiangsu Province}

Next, this paper will analyze the data of Jiangsu province in the process of COVID-19 prevention and control (data source: China COVID-19 Dynamic Table). In order to better reflect the performance of Jiangsu province during the anti-epidemic period, four indicators were summarized and counted, mainly including the rate of new cases, cumulative cases and the quality of change of average population migration.

Among them, the growth rate of new cases reflects the epidemic trend of Novel Coronavirus in Jiangsu province, while the cumulative percentage of cases demonstrates the degree of epidemic prevention and control. Finally, the rate of change of average population migration is a good indicator of the speed at which a region recovers its normal economic activities, which will be calculated in two stages. The data used in the first two lines of this paper were reported from Jiangsu Province, from the first case of COVID-19 to the first day of zero increase in confirmed cases. While the last two lines are from the first COVID-19 case reported in the province (January 22) to the first level response release date (February 24), and the first level response release date (February 24) to the second level response release date (March 27).

Table3. Epidemic prevention and control performance of Jiangsu Province

\begin{tabular}{|l|l|}
\hline & $\begin{array}{l}\text { Jiangsu } \\
\text { Province }\end{array}$ \\
\hline Rate of new cases & $2.03 \%$ \\
\hline Rate of cumulative cases & $39.98 \%$ \\
\hline $\begin{array}{l}\text { Rate of change of average } \\
\text { population migration (during the } \\
\text { first level response period) }\end{array}$ & $44.56 \%$ \\
\hline $\begin{array}{l}\text { Rate of change of average } \\
\text { population migration (during the } \\
\text { second level response period) }\end{array}$ & $431.18 \%$ \\
\hline
\end{tabular}

From the table above, it is not difficult to see that the novel coronavirus has been more obviously controlled in Jiangsu Province, which shows no significant fluctuation trend in the case growth rate. After releasing the first-level response in Jiangsu province, the migrant population moved in quickly, with an average daily growth rate of up to 400 percent.

\section{GENERAL SITUATION AND EPIDEMIC PREVENTION AND CONTROL PERFORMANCE OF SHANDONG PROVINCE}

\subsection{General situation of the development of Shandong Province}

With an area of 150,000 square kilometers and a permanent resident population of 100 million, Shandong is also an economic and populous province in China's coastal areas. Its total GDP in 2019 ranked third in China, second only to Jiangsu and Guangdong provinces. The vast land area, excellent coastal geographical location, large population, and good industrial base make a famous saying: "The development of China reflects in Guangdong in the 1980s, Pudong in the 1990s and Shandong in the early 21st century." This reflects the economic development trend of Shandong, which is favored by many people. Similarly, when the author reviews the literature in the 1990s and the 2010s, the domestic academic circles were widely optimistic about Shandong's economic model, which was led by the government and led by large state-owned enterprises. The public agreed that there would be at least 6-10 years of the rapid growth period, so many analysis reports and related papers have been written at that time.

For example, scholar Huang Bingzhi believes that the "Shandong model" that maintains rapid economic development has advantages for other provinces to learn from. There are three main types of economic development models in Shandong: a self-accumulation economy based on agriculture, economic linkage effect 
with developed transportation, and high urban modernization [1].

From the perspective of culture, Shandong province is the birthplace of Qi-Lu Culture and the source of the inspiring Confucian culture in China. Its core content emphasizes the doctrine of the mean and the cultural values of loyalty, filial piety, sincerity, trust, etiquette, and righteousness, all of which profoundly shape the character and way of doing things on this land of Shandong. Scholar Xu Liyuan believes that the business concept, business psychology, business culture, and business style of Shandong people are closely related to Confucianism. The relationship between righteousness and profit, which puts righteousness first to lead profit, is essential in Confucianism. Confucius regards "righteousness" as the foundation of a human being's life and doing things, and firmly believes that the pursuit of material interests must be based on a way that follows a moral code. He advocated "Benefits guided by righteousness" and "Profitable benefits guided by righteousness." Afterward, Mencius inherited Confucius' thought of righteousness and benefit and approved "Benefits restricted by righteousness, and Benefits from righteousness" [6]. But at the same time, some scholars pointed out few disadvantages of Confucian culture in the development of Shandong enterprises. For example, through investigation and research, scholars Qi Limei and Chen Guo pointed out that the Confucian ideas of faith, righteousness, thrift, and hard work have promoted the Shandong logistics industry's development. However, its more inheritance and less innovation, and local concepts hindered the further development of the Shandong logistics industry [4].

In a word, Shandong Province is another province with a large economy and population in China, with unique geographical advantages, and the birthplace of Qi-Lu Culture, which plays a vital role in the development of Chinese civilization. The government dominates the financial industry in Shandong Province, and the regional culture influences people to be "loyal, filial and righteous," which makes them more likely to obey the arrangement of the superior department.

\subsection{Epidemic prevention and control performance of Shandong Province}

Thanks to the relatively unified historical culture and political system in Shandong province, compared with Jiangsu Province, the 17 cities in Shandong Province are relatively cooperative and harmonious. This characteristic is demonstrated in the fight against COVID-19. Shandong Province consistently implements the policies and epidemic prevention measures promulgated by the provincial government, showing a relatively centralized government governance model.
Similar to the analysis of the epidemic prevention and control performance of Jiangsu Province, this paper will start with the growth rate of new cases, the cumulative case growth rate and the change rate of average population migration to more clearly reflect the overall epidemic prevention and control level of Shandong Province. The data used in the first two lines of this paper were reported from Shandong Province, from the first case of COVID-19. But the difference is that, in order to facilitate comparative analysis with Jiangsu Province, we adjust the cut-off date of case growth rate statistics in Shandong Province with that of Jiangsu Province, both of which are February 24, 2020. While the last two lines are from the first COVID-19 case reported in the province (January 20) to the first level response release date (March 8), and the first level response release date (March 8 ) to the second level response release date (May 5).

Table4. Epidemic prevention and control performance of Shandong Province

\begin{tabular}{|l|l|}
\hline & Shandong Province \\
\hline Rate of new cases & $301.00 \%$ \\
\hline Rate of cumulative cases & $41.0 \%$ \\
\hline $\begin{array}{l}\text { Rate of change of average } \\
\text { population migration (during } \\
\text { the first level response period) }\end{array}$ & $23.01 \%$ \\
\hline $\begin{array}{l}\text { Rate of change of average } \\
\text { population migration (during } \\
\text { the second level response } \\
\text { period) }\end{array}$ & $-2.75 \%$ \\
\hline
\end{tabular}

As can be seen from the comparative analysis of the above data, the growth rate of new cases and cumulative cases in Shandong Province is relatively high, especially the growth rate of new cases has reached a staggering $300 \%$, which shows that the spread of new coronavirus in Shandong Province is relatively fast. The change rate of population migration during the two different levels of prevention and control response in the last two lines that the personnel exchanges in Shandong province are not very large, and the recovery signs of economic activities are not very obvious. The most important is that the average daily rate of population migration showed negative growth in the nearly two months after the reduction to the second-level response. The data mentioned above all reflect the loopholes and deficiencies in preventing and controlling the epidemic in Shandong Province.

\section{CONCLUSION}

Jiangsu Province and Shandong Province, both of which are economically and populous provinces in 
China's coastal areas, are equally ranked in terms of GDP, one ranking second and the other third. The difference lies in the economic development path and pattern of the two. Besides, there are also significant differences in the history and culture of each city within the province. Each city in Jiangsu Province has its characteristics, with various food culture, folk customs, and economic structure. Flowers of every kind are in bloom. However, there is only a single Qi-Lu Culture circle in Shandong province, and the economic model is relatively unified. The large enterprises lead the development modeled by state-owned enterprises, and large enterprises conducted by the government is called "elephant economy" in the academic field.

In terms of the performance of preventing and controlling the spread of new coronavirus at the

Table5. Comparison of the overall level of prevention and control in Shandong and Jiangsu Provinces

\begin{tabular}{|l|l|l|}
\hline & Shandong Province & Jiangsu Province \\
\hline Rate of new cases & $301.00 \%$ & $2.03 \%$ \\
\hline Rate of cumulative cases & $41.0 \%$ & $39.98 \%$ \\
\hline $\begin{array}{l}\text { Rate of change of average } \\
\text { population migration (during the } \\
\text { first level response period) }\end{array}$ & $23.01 \%$ & $44.56 \%$ \\
\hline $\begin{array}{l}\text { Rate of change of average } \\
\text { population migration (during the } \\
\text { second level response period) }\end{array}$ & $-2.75 \%$ & $431.18 \%$ \\
\hline
\end{tabular}

Perhaps the comparison in the previous table is not apparent enough. This time, it will be clearer if we put the two provinces and two utterly different prevention and control practices together. In this contrast, the gap between the two can be clearly reflected. All four indicators of Jiangsu Province are better than those of Shandong Province. In terms of the growth rate of new cases, Shandong Province is $280 \%$ higher than Jiangsu Province; meanwhile, the cumulative increase in cases in Shandong province is also relatively high. In terms of the change rate of population migration, after the release of the first and second level response, the population migration in Shandong Province is slow, and even the population migration has negative growth during the secondary response period, which means that the normalization of economic activities will be delayed. For Jiangsu Province, in turn, after the first and second level prevention and control response was released, the population moved in rapidly, and the recovery of economic activities was also relatively obvious. It can be said that these eight indicators show that the epidemic prevention and control mode of Jiangsu beginning of this year, there are also significant differences between Jiangsu Province and Shandong Province. Shandong province coordinates resources, human resources, and material resources uniformly and always implement the policy documents and the different health QR code issued by the provincial government. The situation in Jiangsu Province is quite the opposite. In contrast, the 13 cities in Jiangsu Province each have their own policies and independent health QR codes. During the nationwide aid to Hubei Province, Jiangsu became famous on the Internet for its "bulk" assistance. These two kinds of government governance modes and response methods can be described as typical cases of flat and vertical government governance models.

province is not only better than that of Shandong province but also has a significant gap.

For China, the world's second-largest economy in terms of GDP, how to further strengthen government governance and efficiency, and what kind of government management mode will help China better adapt to future development and build a well-off society in an all-round way are all fundamental propositions. Since the Third Plenary Session of the 18th CPC Central Committee, the main contents of various successive decisive documents have focused on several key points, such as resource allocation, deconstruction of power consolidation, and innovative development concepts. It can be said that the goals are precise, but it still needs time to form a relatively complete Chinese style innovation theoretical system.

This paper starts with COVID-19 prevention and control performance in two major provinces in China, compares the decentralized and centralized government governance models, the study which mode is more beneficial in coping with the crisis, and makes practical 
explorations. Although this is just an ordinary case analysis, this new perspective can provide a new way of thinking for future social governance and academia. Contrary to what most people think, Jiangsu province, known for its independence and infighting, has far exceeded Shandong Province's overall response to the outbreak of COVID-19, a model of vertical governance that has been given the halo of "being more favorable in responding to public emergencies." In summary, it is shown that the flat government, that is, the multi-level and less subordinate model of governance, is even better than the vertical governance mode in dealing with the sudden public health security.

\section{REFERENCES}

[1] Huang Bingzhi. (1999). The enlightenment of "Shandong Model" on China's economic development. International Business (Journal of University of International Business and Economics) (06), 4-7.

[2] Li Yichun. (2014). Flattening and Social Governance System Innovation. Journal of Qingdao Administration College, Party School of the Qingdao Municipal Committee of the Communist Party of China.

[3] Miao Kai, Hu Jing. (0). Cultural interpretation of the development of Southern Jiangsu. Special Economic Zone (10), 50-52.

[4] Qi Limei, \& Chen Guo. (2015). The Development of Logistics industry in Shandong from the Perspective of Confucian Culture. The Age of Wisdom and Wealth, 000(01X), P.27-.

[5] Wu Baiyi. (0). China should learn from the system of flat social governance in developed countries. China and the World Watch (2015 Issue 3, 4, Issue 39).

[6] Xu Liyuan. (2017). Confucian culture and the business culture of Shandong merchants. Business Culture (20), 16-19.

[7] Zhou Quan. (2009). Standardizing the relationship between vertical management departments and local governments. Operation Managers (18), 176176. 